NORDITA 94/69

gr-qc/9412053

\title{
Dark matter and non-Newtonian gravity from General Relativity coupled to a fluid of strings
}

\author{
Harald H. Soleng*† \\ NORDITA, Blegdamsvej 17, DK-2100 Copenhagen Ø, Denmark
}

(September 27, 1993; revised August 18, 1994)

\begin{abstract}
An exact solution of Einstein's field equations for a point mass surrounded by a static, spherically symmetric fluid of strings is presented. The solution is singular at the origin. Near the string cloud limit there is a $1 / r$ correction to Newton's force law. It is noted that at large distances and small accelerations, this law coincides with the phenomenological force law invented by Milgrom in order to explain the flat rotation curves of galaxies without introducing dark matter. When interpreted in the context of a cosmological model with a string fluid, the new solution naturally explains why the critical acceleration of Milgrom is of the same order of magnitude as the Hubble parameter.
\end{abstract}

PACS numbers: $04.20 .-\mathrm{q} \quad$ 98.80.-k

To appear in the Journal of General Relativity and Gravitation

*E-Mail address: Soleng@surya11.cern.ch

†Present address: Theory Division, CERN, CH-1211 Geneva 23, Switzerland 


\section{INTRODUCTION}

According to the string paradigm the fundamental building blocks of nature are extended objects of dimension one. It is therefore important to understand the gravitational fields produced by a collection of strings rather than point particles. In this vein the concepts of dust clouds and perfect fluids have been extended to string clouds [1] and perfect string fluids [2]. The string-dominated universe model discussed by Vilenkin [3] corresponds to a universe filled with a string cloud. Another approach has been to find exact solutions of gravitational field equations in the presence of a finite number of strings. Examples are the solution for a straight cosmic string [4.5], a solution for two moving straight strings [6], and a solution for $N$ straight strings moving on a circle [7].

Letelier [2] found exact integral expressions for the general solution to Einstein's field equations coupled to a fluid of strings with spherical symmetry. Here we shall consider a static, spherically symmetric string fluid where the transverse pressure is proportional to the energy density. In this case, we are able to give explicit expressions for the exact solution. It is found that a point particle surrounded by a string fluid with a small transverse pressure produces a gravitational field corresponding to a short distance $1 / r^{2}$ and a long distance $1 / r$ force law. As in the vacuum case, the solution is singular at the origin, but this does not affect the validity of the solution as a model of the gravitational field at large $r$.

As an application of the new solution, a string fluid cosmology is presented. In this model the universe is filled with a net of strings. It is assumeed that these strings are pulled out of the net by galaxies and become nearly radially threaded through the galaxies. It is argued that this hypothetical scenario can explain the flat rotation curves of galaxies.

\section{FLUID OF STRINGS}

For the sake of completeness a short review of the results of Letelier [1,2] is given in this section. Just as we have the four-velocity, $u^{\mu}$, associated with the world line of a particle, we have the bivector, $\Sigma^{\mu \nu}$, associated with the world sheet of a string. Let the string world sheet be coordinated by $\lambda^{A} \in\left\{\lambda^{0}, \lambda^{1}\right\}$, where $\lambda^{0}$ and $\lambda^{1}$ are timelike and spacelike coordinates, respectively. Then the world sheet forming bivector is given as

$$
\Sigma^{\mu \nu}=\epsilon^{A B} \frac{\partial x^{\mu}}{\partial \lambda^{A}} \frac{\partial x^{\nu}}{\partial \lambda^{B}}
$$

where $\epsilon^{A B}$ is the antisymmetric, two-dimensional Levi-Civita symbol.

The energy-momentum tensor for a dust cloud is given as $T_{c}^{\mu \nu}=\rho u^{\mu} u^{\nu}$ where $u^{\mu}$ is the normalized four-velocity $\left(u^{\mu} u_{\mu}=-1\right)$ of a dust particle. Similarly, for a string cloud one defines [1]

$$
T_{c}^{\mu \nu}=\rho \sqrt{-\gamma} \Sigma^{\mu \lambda} \Sigma_{\lambda}^{\nu} /(-\gamma)
$$

where $\Sigma^{\mu \nu}$ is the world sheet bivector of each string and where

$$
\gamma \equiv \frac{1}{2} \Sigma^{\mu \nu} \Sigma_{\mu \nu} .
$$


In any astronomical system the symmetries which hold approximatly on a macroscopic scale are broken on a microscopic scale. Even though the universe is isotropic on large scales the velocities of the individual galaxies do not in general conform with the rest frame of the cosmic microwave background. Phenomenologically this situation can be described by regarding each galaxy as a particle in a cosmic fluid. In this model the pressure is a measure of the microscopic deviation from the perfect symmetry [8]. For a general perfect fluid the four-velocity is an averaged four-velocity. In the same way as pressure is introduced for a conventional fluid, one can add a stress tensor proportional to the projection tensor that projects any direction into the space perpendicular to the surface spanned by the averaged bivector $\Sigma^{\mu \nu}$. Let this projection tensor be given by $-\Sigma^{\mu \lambda} \Sigma_{\lambda \nu} / \gamma+\delta^{\mu}{ }_{\nu}$, and let $q$ represent the "pressure" of the string fluid. Then, the energy-momentum tensor for a perfect fluid of strings is [2]

$$
T_{f}^{\mu \nu}=(q+\sqrt{-\gamma} \rho) \Sigma^{\mu \lambda} \Sigma_{\lambda}^{\nu} /(-\gamma)+q g^{\mu \nu}
$$

\section{EXACT SOLUTION}

Consider a static, spherically symmetric space-time. We may write the metric as

$$
d s^{2}=-e^{2 \mu} d t^{2}+e^{2 \lambda} d r^{2}+r^{2} d \theta^{2}+r^{2} \sin ^{2} \theta d \phi^{2}
$$

where $\mu$ and $\lambda$ depend on the radial coordinate $r$, only. The symmetries of this metric restrict $\Sigma_{\mu \nu}$ to have only two nonvanishing components, $\Sigma_{t r}$ and $\Sigma_{\theta \phi}$. Physically this means that the averaged world sheets are either in the $(r, t)$ or $(\theta, \phi)$-planes. The condition $\gamma<0$ (the world sheets are not space-like) and the definition (2.1), tell us that only the $\Sigma_{t r}$ component survive [回]. This means that the averaged world sheets are in the $(t, r)$-plane, but only in the exceptional case of a string cloud does the individual strings orient themselves in a perfect radial fashion. Thus, the energy-momentum tensor (2.4) of a perfect string fluid, reduces to

$$
T_{t}^{t}=T_{r}^{r} \text { and } T_{\Omega}^{\Omega}=q
$$

where $\Omega$ stands for both $\theta$ and $\phi$.

The Ricci tensor for the line-element (3.1) takes the form

$$
\begin{aligned}
& R_{t}^{t}=\left(-\mu^{\prime \prime}+\lambda^{\prime} \mu^{\prime}-\mu^{\prime 2}-2 \frac{\mu^{\prime}}{r}\right) e^{-2 \lambda} \\
& R_{r}^{r}=\left(-\mu^{\prime \prime}+\lambda^{\prime} \mu^{\prime}-\mu^{\prime 2}+2 \frac{\lambda^{\prime}}{r}\right) e^{-2 \lambda} \\
& R_{\Omega}^{\Omega}=\left(-\frac{\mu^{\prime}}{r}+\frac{\lambda^{\prime}}{r}-\frac{1}{r^{2}}\right) e^{-2 \lambda}+\frac{1}{r^{2}} .
\end{aligned}
$$

The gravitational field is uniquely determined once an "equation of state" specifies the relation between $T_{t}^{t}$ and $T_{\Omega}^{\Omega}$. Here we shall assume that $T_{t}^{t}{ }_{t}$ is proportional to $T_{\Omega}^{\Omega}$. Thus 
we get an energy-momentum tensor of the form円

$$
T_{t}^{t}=T_{r}^{r}=-\alpha T_{\Omega}^{\Omega}
$$

where $\alpha$ is a dimensionless constant. Using geometrized units, i.e. $G=c=1$, and the above energy-momentum tensor, Einstein's field equations

$$
G^{\mu}{ }_{\nu} \equiv R_{\nu}^{\mu}-\frac{1}{2} g^{\mu}{ }_{\nu} R=8 \pi T_{\nu}^{\mu}
$$

imply

$$
G_{t}^{t}=G_{r}^{r} \quad \text { and } \quad G^{t}{ }_{t}=-\alpha G_{\Omega}^{\Omega}
$$

From the first equation we find $\lambda=-\mu$. Then the second equation becomes

$$
\frac{1}{r^{2}}\left[\left(r e^{2 \mu}\right)^{\prime}-1\right]=-\frac{\alpha}{2 r}\left[\left(r e^{2 \mu}\right)^{\prime}-1\right]^{\prime} .
$$

The case $\alpha=0$ corresponds to the Schwarzschild solution. For $\alpha \neq 0$, integration yields

$$
\left(r e^{2 \mu}\right)^{\prime}-1=-\varepsilon\left(\frac{\ell}{r}\right)^{2 / \alpha}
$$

where $\ell$ is a positive integration constant of dimension length and $\varepsilon= \pm 1$ is a sign factor which determines the sign of the energy-density of the string fluid ( $\varepsilon=1$ corresponds to a positive energy density). Integrating once more, we find

$$
e^{2 \mu}=1-\frac{2 M}{r}- \begin{cases}\varepsilon \ell r^{-1} \ln (\lambda r) & \text { for } \alpha=2 \\ \varepsilon \alpha(\alpha-2)^{-1} \ell^{2 / \alpha} r^{-2 / \alpha} & \text { for } \alpha \neq 2 .\end{cases}
$$

This is the general solution for static and spherically symmetric space-times with a radially boost invariant energy-momentum tensor with a constant equation of state. The following special cases are singled out: $\alpha=-1$ corresponds to the Schwarzschild-de Sitter solution, $\alpha=0$ gives the Schwarzschild solution, and $\alpha=1$ represents the Reissner-Nordström solution. For the case $\alpha=\infty$, the correction term in the metric coefficient of Eq. (3.9) is a constant, and thus in the zero temperature limit the strings do not produce any gravitational forces. This result agrees with Letelier's [1] solution for a static cloud of strings with spherical symmetry. In this case the strings are exactly straight and radially directed. A straight ideal string has vanishing gravitational mass, because the gravitational effect of tension exactly cancels the effect of its mass [4.5]. The only remaining gravitational effect is an angle

\footnotetext{
${ }^{1}$ Energy-momentum tensors of this algebraic form (also with a variable $\alpha$ ) were first considered by Petrov [9], and later discussed by Gliner [10]. Dymnikova [11] and Soleng [12] have recently interpreted it as the energy-momentum tensor associated with anisotropic vacuum polarization in a spherically symmetric space-time.
} 
deficit. The same happens in the spherically symmetric solution, but here there is a solid angle deficit.

In the generic case, $\alpha \in\langle-\infty, \infty\rangle \backslash\{0,2\}$, the classical gravitational acceleration is

$$
g=\frac{M}{r^{2}}+\varepsilon \frac{\ell^{-1}}{(\alpha-2)}\left(\frac{\ell}{r}\right)^{1+2 / \alpha} .
$$

The energy-density, $\rho_{s}$, corresponding to these solutions are found from Eqs. (3.7) and (3.8). Hence, using that $8 \pi \rho_{s}=-8 \pi T^{t}{ }_{t}=-G^{t}{ }_{t}$, one finds

$$
8 \pi \rho_{s}=\frac{\varepsilon}{r^{2}}\left(\frac{\ell}{r}\right)^{2 / \alpha}
$$

The divergence of $T^{\mu}{ }_{\nu}$ as $r \rightarrow 0$ signals that the model breaks down at small distances. The singularity at the origin is not unique for the string fluid model: also the Schwarzschild solution of a point mass in vacuum is a singular space-time, and the Reissner-Nordström solution of a charged point mass has a diverging energy-momentum tensor at the origin. The singularities at the origin do not invalidate these solutions as models of the gravitational field outside masses with charges or masses surrounded by a string fluid.

\section{DARK MATTER OR MODIFIED GRAVITY}

Einstein's theory has had remarkable success in explaining observed and inferred gravitational phenomena. There seems to be only one serious problem - the missing mass problem. On large scales, the scales of galaxies and beyond, the Einstein-Newton dynamics seems to imply that there is much more mass than we can observe directly. From observations of the rotational velocities of the gaseous component of galaxies, it is found that the velocity approaches a constant at large distances [13], and from the relation $v^{2} / r=g$, one finds that the gravitational acceleration decreases as $1 / r$ here. According to Newtonian gravity, this corresponds to an effective mean mass density $\rho(r) \sim 1 / r^{2}$ and a total mass increasing linearly with distance. If luminuous matter were a good tracer of mass, and if Newton's law were valid at these scales, one should find $g \sim 1 / r^{2}$ and a mean mass density of $\rho(r) \sim 1 / r^{3}$ corresponding to a constant total mass at large distances.

There are three explanations to this discrepancy: (a) Newtonian dynamics is wrong at these scales, $(b)$ there is a lot of unseen "dark matter" in the galaxies, or $(c)$ non-gravitational forces play an important rôle in the motion of the gaseous component of galaxies, e.g. magnetic fields [14]. Many authors have advocated the first explanation, and modifications of Newton's gravitational dynamics have been proposed [15]20]. Milgrom's theory (for reviews, see Refs. [21 23]) has worked impressingly well both for galaxies [24 26] and galaxy systems [27], and Begeman et al. [28] have claimed that it gives the best phenomenological description of the systematics of the mass discrepancy in galaxies. Others come to a different conclusion, cf. Ref. [29] (see also Milgrom's rebuttal [30]) and Ref. [31]. Another argument

in favor of an effective $1 / r$ correction to the force law at large distances, is that such a term could stabilize a cold stellar disk in a numerical galaxy model [32]. But this would happen also if the $1 / r$ effective force law was due to dark matter. 
In spite of the phenomenological success of non-Newtonian dynamics, the scientific community has been reluctant to abolish Newton's theory of gravitation, partly because Newton's theory is far more aesthetically attractive than any of the modified theories, and since the interactions appear to be more fundamental than matter, one would rather introduce new matter than new forces, but above all it is objected that none of the modified theories have a viable relativistic counterpart [23,33]. The attempts in this direction, the aquadratic Lagrangian theory [17, and phase coupling gravitation [34.35] have lead to tachyonic propagation [36] or problems with gravitational lensing measurements [37], respectively, and it seems that if a viable scalar-tensor theory that mimics Milgrom's exists, it will be very complicated and contain many new fundamental constants. Therefore, the most widely accepted explanation is that the universe is filled with huge amounts of dark matter.

From Eq. (3.10) one gets an effective attractive 1/r correction to Newton's force if $|\alpha| \gg$ 1. It is therefore tempting to propose a string fluid as a specific form of dark matter. In the case of spherical symmetry, this energy-momentum tensor reproduces a force law similar to Milgrom's [16] within the framework of General Relativity 38.

\section{STRING FLUID BACKGROUND AND DARK MATTER}

\section{A. String fluid cosmology}

It has earlier been proposed that dark matter on cosmic scales may be composed of strings [3]. A good review of the physics of cosmic strings can be found in Ref. [39]. The astronomical constraints on a string-dominated universe have been analyzed by Gott and Rees [40]. They concluded that if the strings have a mass per unit length below $G \mu \approx 10^{-14}$ $10^{-15}$, the strings could contribute with more than five times the critical density and still be in agreement with observational data. If the mass per length is very small, such as $G \mu \approx 10^{-30}$, then the strings would be difficult to detect even when passing through the observer [3]. A string-dominated universe requires that the intercommuting probability of the strings is very small, i.e., the strings pass freely through one another. In contrast, simulations of string models have lead to a high intercommuting probability. On account of this, string-dominated universe models have lost favour among theorists. Needless to say, the evolution of a string network is model dependent, and just as one has very weakly interacting particles, it is a priori possible to have very weakly interacting strings. Hence, there is no fundamental principle which forbids a string-dominated universe. Also, astronomical data are compatible with this hypothesis. Admittedly, it could be discussed how natural this hypothesis is, but for the moment we adopt it as a working hypothesis in order to see where it leads.

An isotropic stringy background (a string cloud) has an energy-density of the form [39]

$$
8 \pi \rho_{s}=\frac{3 w}{R^{2}}
$$

where $R$ is the cosmic scale factor and $w$ is a constant. Accordingly, the first Friedmann equation takes the form

$$
H^{2}=\frac{8 \pi}{3} \rho_{m}+\frac{w-k}{R^{2}}
$$


where $\rho_{m}$ represents the non-stringy energy density. This means that if one neglects the stringy background, the effective curvature is $k_{\text {eff }}=k-w$ rather than $k$. In this way a closed universe with a geometric $k \approx 0$ as predicted by inflation could be in agreement with the observed $k_{\text {eff }}<0$, if the universe is filled with a string cloud [39].

\section{B. String fluid in galaxies}

The hypothesis that the universe is filled with a string fluid where each string has a mass density below what is directly observable, can only be tested by the effect of the string fluid on the local gravitational fields of galaxies. On the other hand the gravitational fields of galaxies are expected to affect the motion of strings. According to the equivalence principle all masses fall in the same fashion. In general, due to tidal forces, elongated objects will tend to orient themselves along the gravitational field lines. This could provide a mechanism whereby strings are pulled out of the cosmic ensemble by every galaxy and redirected so as to appear approximately radially oriented near each galaxy. If the string tension is not too large, it is possible that a significant number of strings are trapped by the galaxies. Such stringy halos could produce a measureable change in the long range gravitational fields of the galaxies.

The cosmic string fluid is assumed to be cold. Strings which are trapped by galaxies would be heated due to release of potential gravitational energy. Let a string segment of length $R_{s}$ be trapped in this way. The potential gravitational energy inside a region of radius $R_{s}$ is

$$
E_{p}=-\frac{M m_{s}}{R_{s}^{2}}
$$

where $M$ is the mass of the galaxy and where $m_{s}=\mu R_{s}$ is the mass of the trapped string segment. If the string was trapped in the early universe and if the released energy flows along the string with the speed of light, it would now be distributed over at most one Hubble length, $H_{0}^{-1}$. Had the string been trapped later, this length would have been shorter. Let us, however, consider the Hubble length which is relevant if most of the strings were trapped before or at the time of galaxy formation. The fraction $R_{s} H_{0}$ of $-E_{p}$ remains in the trapped part of the string. Thus, this part of the string could gain an energy per mass of

$$
\frac{\Delta E}{m_{s}} \approx-\frac{E_{p} R_{s} H_{0}}{m_{s}}=M H_{0}
$$

by falling into a galaxy of mass $M$. This energy is available to produce transverse motion of the string. For the string fluid this leads to a transverse pressure given by

$$
(p V)^{2}=\left(m_{s}+\Delta E\right)^{2}-m_{s}^{2} .
$$

The transverse pressure will contribute with a pressure per energy density $p V / m_{s} \approx$ $\left(M H_{0}\right)^{1 / 2}$. This determines the dimensionless constant in Eq. (3.4) as follows

$$
\alpha \approx\left(M H_{0}\right)^{-1 / 2}
$$


where we have used that the string fluid energy-density and transverse pressure are positive to conclude that $\varepsilon=1$. The string fluid contributes with a positive effective gravitational mass. This agrees with the General Relativity lore that pressure contributes to gravitational attraction. The value of $\alpha$ in Eq. (5.6) is large. A large $\alpha$ corresponds to $\left|T_{t}^{t}\right|=\left|T_{r}^{r}\right| \gg$ $\left|T_{\Omega}^{\Omega}\right|$. Physically, the solution (3.9) with a large $\alpha$ represents a fluid of nearly radially directed strings at low but nonzero temperature. Recall that the $\alpha \gg 1$ solution implies an $1 / r$ correction to Newton's gravitational force law. This is what is needed to explain the flat rotation curves of galaxies. The term $\ell^{2 / \alpha}$ in Eq. (3.10) is an a priori arbitrary normalization constant, that determines the density of strings or, equivalently, normalizes the energy density of the string fluid. From a mathematical perspective $\ell^{2 / \alpha}$ is a free parameter, but from a physical point of view, certain values are more reasonable than others: $\ell$ is a constant of dimension length and in a fundamental underlying theory it has to be determined by physical constants. The smallest and largest units of length we can think of are the Planck length and the Hubble length, and in my opinion it is reasonable to assume that $\ell$ is within this range. The predicted value of $|\alpha|$ is so large that $(\ell / r)^{2 / \alpha} \approx 1$ for both the extreme possibilities for $\ell$. Hence, with this information, Eq. (3.10) implies that Newton's force law is changed to

$$
g=\frac{M}{r^{2}}+k_{0} \frac{\left(M H_{0}\right)^{1 / 2}}{r}
$$

where the constant $k_{0} \approx 1$. The presence of the Hubble parameter in the local force law signals that the translational invariance of the background string fluid is broken not only in spatial directions but also in the time-direction.

Note that the force law of Eq. (5.7) agrees with the Tully-Fisher law [41] which relates the rotational velocity, $v$, to the luminosity, $L$, by $v \propto L^{1 / 4}$ if the luminosity is proportional to the Newtonian mass. This is a reasonable assumption if the ratio of dark and luminous matter densities is a constant. Also, the mysterious coincidence that Milgrom's critical acceleration is equal to the Hubble parameter [16,23], is explained as a result of having dark matter of cosmic extension.

\section{DISCUSSION}

An exact static, spherically symmetric solution of the field equations of General Relativity coupled to a string fluid with a constant equation of state has been found. For the same space-time symmetries, this is also the most general solution for any radially boost invariant energy-momentum tensor with a constant equation of state. The solution is singular at the origin. This singularity can be attributed to the high degree of symmetry of the model, and does not affect the behaviour at large $r$. It has been observed that the solution reproduces a force law similar to Milgrom's [16]. This motivates a string model for dark matter. According to this model, the new solution can explain the Machian character of Milgrom's acceleration, $a_{0} \approx H_{0}$. The dark matter model presented here is a phenomenological model, but it can no longer be objected that the $1 / r$ modification of Newton's force law is "an orphan in the classical world" [33]. Instead it follows from General Relativity with an energy-momentum tensor corresponding to a string fluid provided this string fluid satisfies the equation of state 
$T^{t}{ }_{t}=-\alpha T_{\Omega}^{\Omega}$ where $\alpha \approx\left(M H_{0}\right)^{-1 / 2}$. Reasonable assumptions concerning the infall of strings into galaxies have lead to this value for $\alpha$.

Up to now the missing mass problem has been resolved by assuming that dark matter is present in whatever quantities and distributions that are needed to explain away all mass descrepancies. The main problem with this approach is that the dark matter hypothesis in this form is too flexible to give any unavoidable predictions [23], and it is in principle not testable before one specifies the nature of the dark matter. In contrast, the approach of Milgrom is testable, and it gives specific predictions which are in good agreement with observations. The main problem has been that the modified dynamics has had no viable relativistic counterpart.

Despite the success of Milgrom's $1 / r$ force law and the fact that the present model reproduces it, there are many reasons why it is premature to identify a string fluid as the solution to the missing mass problem. First, there is no field theoretic realization of this particular model. Second, the value $\alpha \approx\left(M H_{0}\right)^{-1 / 2}$ has been found only with the additional assumption that the potential gravitational energy of the strings has been spread over a length of $H_{0}^{-1}$. On the other hand, it is natural to suspect that an isotropic background of strings which only results in a redefinition of the effective spatial curvature on cosmic scales, only contributes with a similar redefinition of the effective asymptotic space in the geometry of isolated masses. Then, only the gravitationally trapped strings, i.e., those described by the energy-momentum tensor of the solution, are relevant for local gravitational field, and the solution is in fact unique up to a specification of $\alpha$. For $\alpha^{-1}$ which is zero in the string cloud of the cosmic background, one would expect only a small perturbation near galaxies. The perturbation away from $\alpha^{-1}=0$ would be expected to depend on the two characteristic length scales of the problem, namely the Hubble parameter and the mass of the galaxy. This is just the result obtained by an energy argument. To clarify this issue one needs to solve the embedding problem where the local solution is embedded in a string-dominated universe. Third, the proposal has only been studied in a static, spherically symmetric model. In contrast, the real universe is non-static and it contains many galaxies, so both of the symmetry assumptions are broken. The consequences of deviations from spherical symmetry are not clear, and it is also a question how strings passing through more than one galaxy will affect the model. Finally, one expects realistic string models to predict that strings will intercommute and produce closed loops which collapse and leave the universe with very few strings.

In spite of these problems, from a general relativistic perspective, it is very interesting that such a simplistic model can reproduce the non-Newtonian force law. The generally accepted solution - dark matter - need therefore not be a very complicated system of epicycles as have been argued by the proponents of non-Einsteinian gravitation, e.g. Sanders [19].

\section{Acknowledgements}

It is a pleasure to thank Paul J. Steinhardt for stimulating my interest in the dark matter problem and for valuable comments. I am also grateful to Jacob Bekenstein and Mordehai Milgrom for correspondence and critical remarks, and I thank Øyvind Grøn for carefully reading through the manuscript. Thanks are also due to the referees who have contributed with constructive criticism and a number of references to the literature. This research was 
initiated at the University of Pennsylvania where I spent my sabbatical year 1992-93 on leave from from the University of Oslo. I acknowledge support from the Thomas Fearnley Foundation, from Lise and Arnfinn Heje's Foundation, Ref. No. 0F0377/1993, and from the U.S. Department of Energy under Contract No. DOE-EY-76-C-02-3071. 


\section{REFERENCES}

[1] Letelier, P. (1979). Phys. Rev. D 20, 1294.

[2] Letelier, P. (1981). Il Nuovo Cimento 63 B, 519.

[3] Vilenkin, A. (1984). Phys. Rev. Lett. 53, 1016.

[4] Gott, J. R. (1985). Astrophys. J. 288, 422.

[5] Hiscock, W. A. (1985). Phys. Rev. D 31, 3288.

[6] Gott, J. R. (1991). Phys. Rev. Lett. 66, 1126.

[7] Kabat, D. N. (1992). Phys. Rev. D 46, 2720.

[8] Soleng, H. H. (1990). Astron. Astrophys. 237, 1.

[9] Petrov, A. Z. (1969). Einstein Spaces, (Pergamon Press, Oxford).

[10] Gliner, É. B. (1966). Sov. Phys. JETP 22, 378.

[11] Dymnikova, I. (1992). Gen. Rel. Grav. 24, 235. 235.

[12] Soleng, H. H. (1994). Gen. Rel. Grav. 26, 149.

[13] Sancisi, R., and van Albada, T. S. (1987). In Dark Matter in the Universe, 117th IAU symposium, p67, eds. Kormendy H., and Knapp, G. R., (D. Reidel Publishing Co., Dordrecht, Holland).

[14] Battaner, E., Garrido, J. L., Membrado, M., and Florido, E. (1992). Nature 360, 652.

[15] Finzi, A. (1963). Mon. Not. Roy. Astr. Soc. 127, 21.

[16] Milgrom, M. (1983). Astrophys. J. 270, 365.

[17] Bekenstein J., and Milgrom, M. (1984). Astrophys. J. 286, 7.

[18] Kuhn J. R., and Kruglyak, L. (1987). Astrophys. J. 313, 1.

[19] Sanders, R. H. (1990). Astron. Astrophys. Rev. 2, 1.

[20] Liboff, R. L. (1992). Astrophys. J. Lett. 397, L71.

[21] Milgrom, M. (1987). In Dark Matter in the Universe: proceedings of the 4th Jerusalem Winter School for Theoretical Physics, p231, eds. Bahcall, J. N., Piran, T., and Weinberg, S., (World Scientific Publ., Singapore).

[22] Milgrom M., and Bekenstein, J. (1987). In Dark Matter in the Universe, 117th IAU symposium, p319, eds. Kormendy H., and Knapp, G. R., (D. Reidel Publishing Co., Dordrecht, Holland).

[23] Milgrom, M. (1989). Comm. Astrophys. 13, 215.

[24] Milgrom, M. (1983). Astrophys. J. 270, 371.

[25] Milgrom, M. (1984). Astrophys. J. 287, 571.

[26] Milgrom, M. (1986). Astrophys. J. 302, 617.

[27] Milgrom, M. (1983). Astrophys. J. 270, 384.

[28] Begeman, K. G., Broeils, A. H., and Sanders, R. H. (1991). Mon. Not. Roy. Astron. Soc. 249, 523.

[29] Lake, G. (1989). Astrophys. J. 345, L17.

[30] Milgrom, M. (1991). Astrophys. J. 367, 490.

[31] Gerhard, O. E., and Spergel, D. N. (1992). Astrophys. J. 397, 38.

[32] Tohline, J. E. (1983). In Internal Kinematics and Dynamics of Galaxies, 100th IAU symposium, p205, ed. Athanassoula, A., (D. Reidel Publishing Co., Dordrecht, Holland).

[33] Lindley, D. (1992). Nature 359, 583.

[34] Bekenstein, J. (1988). In Proc. of 2nd Canadian Conf. on General Relativity and Relativistic Astrophysics, p68, eds. Coley, A., Dyer, C., and Tupper, T., (World Scientific Publ., Singapore). 
[35] Bekenstein, J. (1988). Phys. Lett. B 202, 497.

[36] Bekenstein, J. (1990). In Developments in general relativity, astrophysics and quantum theory: A jubilee volume in honour of Nathan Rosen, p155, eds. Cooperstock, F., Horwitz, L. P., and Rosen, J., (IOP Publ., Bristol, England).

[37] Bekenstein, J. (1992). In Proceedings of The 6th Marcel Grossman Meeting on General Relativity, p905, eds. Sato, H., and Nakamura, T., (World Scientific Publ., Singapore).

[38] Ishizawa, T. (1987). In Dark Matter in the Universe, 117th IAU symposium, p334, eds. Kormendy H., and Knapp, G. R., (D. Reidel Publishing Co., Dordrecht, Holland) gave another suggestion for dark matter. He argued that a $1 / r$ correction to Newton's force law results from radially arranged flux tubes.

[39] Vilenkin, A. (1985). Phys. Rep. 121, 263.

[40] Gott, J. R. and Rees, M. J. (1987). Mon. Not. Roy. Astr. Soc. 277, 453.

[41] Tully, R. B., and Fisher, J. R. (1977). Astron. Astrophys. 54, 661. 